\title{
Absolutely FABulous? FABP-1 in Non-acetaminophen-related Acute Liver Failure
}

\author{
A. Cavazza ${ }^{1} \cdot$ M. J. McPhail $^{1}$
}

Published online: 23 April 2020

(c) Springer Science+Business Media, LLC, part of Springer Nature 2020

Acute liver failure (ALF) remains a challenging disease to treat. Despite advances in spontaneous and post-transplant survival, a substantial proportion of patients die; decisions on listing for emergency liver transplant (ELT) are dependent on imperfect non-biological clinical criteria [1]. Although there has been considerable scientific effort to improve the prognostic accuracy of listing criteria, the original Kings College Criteria (KCC) [2], published in the 1980s, remain the most widely used despite ongoing concerns over their low sensitivity $[3,4]$. One of the key factors contributing to the sustained use of the KCC has been the size of the original cohort (particularly in the number of patients not undergoing ELT) helping validate the criteria. As such, they remain the benchmark for ELT worldwide.

In the search for novel biomarkers, the work of the US Acute Liver Failure Study Group (USALFSG) is vital and unique in the breadth, size and depth of their cohort and associated biobank. This important group has developed novel scoring systems that in some reports improve prognostic accuracy with or without the addition of novel markers [5].

Previously, Karvellas et al. explored the use of the novel biomarker fatty acid binding protein 1 (FABP-1) in acetaminophen-induced ALF (APAP ALF), finding this to improve on existing prognostic criteria [6], even when covariates such as MELD were included, suggesting that FABP-1 in APAP ALF offers an improvement over the KCC.

FABPs are small cytoplasmic proteins that belong to a family of molecules responsible for the transport of longchain fatty acids. Of the nine forms presently known FABP-1 is primarily expressed in hepatocytes. Elevated levels of urinary FABP-1 can discriminate between patients with

\footnotetext{
M. J. McPhail

mark.mcphail@kcl.ac.uk

1 Liver Intensive Therapy Unit, Institute of Liver Studies, Kings College Hospital, Kings College London, London, UK
}

acute tubular necrosis and hepatorenal syndrome in cirrhotic patients [3]. FABP2 is expressed in intestine, while FABP3-9 is present in other organs. Although originally their mechanistic function was thought to be related to lipid shuttling, more evidence is being collated to suggest that their dysregulation is important in many metabolic diseases such as cardiovascular disease, cancer, obesity and in chronic liver diseases such as nonalcoholic fatty liver disease.

In this issue of Digestive Diseases and Sciences, Karvellas et al. now explore FABP-1 in patients with non-APAP ALF [7]. Given their previous work in APAP ALF and FABP-1, the authors speculate that FABP-1 in non-APAP ALF may similarly correlate with survival. Using a nested case control design using samples from 198 patients in the USALFSG, they found that while increased serum FABP-1 levels present at early and late time points were seen in nonsurvivors, they were not independently associated with mortality on multivariate analysis. FABP- $1>350 \mathrm{ng} / \mathrm{mL}$ was associated with higher risk of death.

The study has a number of important strengths. All samples were measured by ELISA in duplicate and a coefficient of variance $(\mathrm{CV})$ of $<25 \%$ was used to define acceptable variation. Similar to the earlier large cohort studies, the size of the study enables important subgroup classification and validation to be performed. Nevertheless, the long-term stability of FABP-1 was not assessed nor plate-to-plate variability of the assay described. Yet, these are minor criticisms as it appeared clear that despite this attention to detail in measurement protocols, FABP-1 was not behaving in the same manner as with APAP ALF.

While non-APAP ALF is deemed a single group for transplant listing status in the KCC, it is clear that this category encompasses a number of different clinical phenotypes. Patients with hypoxic hepatitis such as 3,4-methylenedioxymethamphetamine (MDMA/ecstasy) or cocaine overdose can behave similarly to APAP cases with hyperacute ALF whereas those with more indolent subacute seronegative 
aetiologies evolve over a more prolonged period. This not only yields a phenotype with higher serum bilirubin and longer time to multi-organ failure, but also less histological hepatic necrosis and a higher risk of sepsis. Therefore, it may not be surprising that the liver-specific FABP-1 was not associated independently with poor outcome. It may therefore be of further interest how FABP-1 is modulated not only in the first week following presentation but in the subsequent weeks before further deterioration or recovery.

Karvellas et al. explored the link between FABP-1 and organ failure and noted that there was correlation between markers of extrahepatic organ failure and FABP-1. This may be an epiphenomenon but is worth further investigation. It would have been of further interest to explore this marker in tandem with well-established markers of hepatic necrosis and apoptosis and cytokine arrays. This further mechanistic exploration may have yielded supporting evidence that FABP-1 is or is not associated with hepatic necrosis.

Do these findings also hint that in non-APAP ALF, other factors are of equal importance to liver failure? Given the strong associations with extrahepatic organ failure and outcome, these results could further strengthen this important clinical observation. Also given the recent interest in the different metabolic, immunological and clinical phenotypes of subacute liver failure, an exploration of intestinal FABP2 could also be of significant interest. While the majority of studies investigating the involvement of the gut-liver axis are in patients with cirrhosis, there is considerable scientific merit in investigating these processes in patients with ALF [8]. FABP2 is primarily of intestinal origin; together with D-lactate or measures of bacterial DNA may be a more fruitful avenue of exploration. Conversely, FABP-1 is not only expressed in the liver but also in enterocytes and in smaller amounts in the kidney. Therefore, the involvement of the gut in non-APAP ALF may further complicate and confound the levels of FABP-1 observed in the serum in the cohort of Karvellas et al.

In summary, while measurement of serum FABP-1 is unlikely to change clinical management of non-APAP ALF, this study represents an important piece of additional evidence to inform the understanding of less hyperacute forms of ALF where hepatic necrosis may not be the only driver of inflammatory responses and extrahepatic processes require further examination.

\section{References}

1. Trovato FM, Rabinowich L, McPhail MJW. Update on the management of acute liver failure. Curr Opin Crit Care. 2019;25:157-164.

2. O'Grady JG, Alexander GJ, Hayllar KM, et al. Early indicators of prognosis in fulminant hepatic failure. Gastroenterology. 1989;97:439-445.

3. McPhail MJ, Wendon JA, Bernal W. Meta-analysis of performance of Kings's College Hospital Criteria in prediction of outcome in non-paracetamol-induced acute liver failure. J Hepatol. 2010;53:492-499.

4. Bailey B, Amre DK, Gaudreault P. Fulminant hepatic failure secondary to acetaminophen poisoning: a systematic review and meta-analysis of prognostic criteria determining the need for liver transplantation. Crit Care Med. 2003;31:299-305.

5. Rutherford A, King LY, Hynan LS, et al. Development of an accurate index for predicting outcomes of patients with acute liver failure. Gastroenterology. 2012;143:1237-1243.

6. Karvellas CJ, Speiser JL, Tremblay M, et al. Elevated FABP1 serum levels are associated with poorer survival in acetaminophen-induced acute liver failure. Hepatology. 2017;65:938-949.

7. Karvellas CJ, Speiser JL, Tremblay M, et al. Elevated serum livertype fatty acid binding protein levels in non-acetaminophen acute liver failure patients with organ dysfunction. Dig Dis Sci. (Epub ahead of print). http://doi.org/10.1007/s10620-020-06166-w.

8. Gong S, Lan T, Zeng L, et al. Gut microbiota mediates diurnal variation of acetaminophen induced acute liver injury in mice. $J$ Hepatol. 2018;69:51-59.

Publisher's Note Springer Nature remains neutral with regard to jurisdictional claims in published maps and institutional affiliations. 